\title{
An inertial iterative scheme for solving variational inclusion with application to Nash-Cournot equilibrium and image restoration problems
}

\author{
Jamilu Abubakar, Poom Kumam, Abor Isa Garba, Muhammad Sirajo \\ ABDUllahi, ABDUlKarim HASSAN IBRAHIM and KANOKWAN SITTHITHAKERNGKIET
}

\begin{abstract}
Variational inclusion is an important general problem consisting of many useful problems like variational inequality, minimization problem and nonlinear monotone equations. In this article, a new scheme for solving variational inclusion problem is proposed and the scheme uses inertial and relaxation techniques. Moreover, the scheme is self adaptive, that is, the stepsize does not depend on the factorial constants of the underlying operator, instead it can be computed using a simple updating rule. Weak convergence analysis of the iterates generated by the new scheme is presented under mild conditions. In addition, schemes for solving variational inequality problem and split feasibility problem are derived from the proposed scheme and applied in solving Nash-Cournot equilibrium problem and image restoration. Experiments to illustrate the implementation and potential applicability of the proposed schemes in comparison with some existing schemes in the literature are presented.
\end{abstract}

\section{INTRODUCTION}

Variational inclusion (VI) problem is a problem of determining a point $u^{*} \in \mathbb{H}$, such that

$$
0 \in\left(A u^{*}+B u^{*}\right)
$$

where $A: \mathbb{H} \longrightarrow 2^{\mathbb{H}}$ is a multi-valued operator and $B: \mathbb{H} \longrightarrow \mathbb{H}$ is a single-valued operator. The problem (1.1) is an important general problem that can be interpreted and modeled as several problems in different areas of research, such as optimization theory, optimal control, transportation problem and so on (Refs. $[8,12,13,17,18,20,23,25,29,30$, $31,34,36,43,59,55,57])$. Applications of variational inclusion problem in solving different real-world problems ranging from compressed sensing, image processing, radiation therapy treatment planning, have led to increasing interest in devising iterative methods for solving problem (1.1).

The popular method for solving (1.1) is the forward-backward splitting method proposed in [37] and [50]. The scheme generates a sequence $u_{n}$ for $n \geq 1$ as follows:

$$
u_{n+1}=(I+\lambda A)^{-1}(I-\lambda B) u_{n},
$$

where $(I+\lambda A)^{-1}$ is the resolvent operator associated with the operator $A$ and $\lambda$ is a positive parameter. It has been shown that, the convergence of (1.2) requires some restrictive assumptions on the underlying operators. For instance, $A$ is required to be cocoercive or $B$ to be inverse strongly monotone operator. In an effort to weaken some of these strong assumptions in (1.2), Tseng in [58] introduced a modified forward-backward method for

Received: 17.08.2020. In revised form: 29.03.2021. Accepted: 11.06.2021

2010 Mathematics Subject Classification. 47H05, 47J20, 47J25, 65K15.

Key words and phrases. variational inclusion problem, inertial step, split convex feasiblity method, Nash equilibrium, image debluring, maximal monotone operator.

Corresponding author: Poom Kumam; poom.kum@kmutt.ac.th 
solving (1.1). Weak convergence of the scheme in [58] is established under the condition that, $A$ is maximal monotone and $B$ is a monotone operator.

Recently, the authors in [41] successfully introduced another modified forward backward method called forward-reflected-backward splitting method which also does not requires the cocoercivity assumption on the operator $B$. Although, the convergence of the modified forward-backward schemes in [58] and [41] are formulated without the strong assumptions, it can be observed that the stepsizes considered in these works are either a fixed stepsize chosen in $\left(0, \frac{1}{L}\right)$ ( $L$ is the Lipschitz constant of $B$ ) or the stepsize can be computed using a line search procedure with finite stopping criterion. It is known that, line search procedures involve extra functions evaluations or computations of resolvent of the considered operator, thereby reducing the computational performance of a given scheme. A modification of the forward-reflected-backward splitting method is proposed in [61] by considering variable stepsizes which are updated over each iteration by some simple computations given as: choose $\lambda_{0}>0$ and $\nu \in\left(0, \frac{1}{2}\right)$ such that

$$
\lambda_{n+1}=\min \left\{\frac{\nu\left\|u_{n+1}-u_{n}\right\|}{\left\|B u_{n+1}-B u_{n}\right\|}, \lambda_{n}\right\} \quad n \geq 1 .
$$

The stepsize (1.3) is updated without the prior knowledge of the Lipschitz constant of the underlying operator. Several variants of the forward-backward-forward method have been studied and a number of schemes have been presented to solve (1.1) (Refs. [1, 6, 19, $23,24,27,32,33,49,52,53,55,58]$ ).

Inertial extrapolation techniques are introduced as a process of accelerating the convergence rate of an iterative scheme. These methods trace back to the pioneering work of Polyak [51] who introduced the heavy ball method to speed up the gradient algorithm's convergence behavior and allow the identification of various critical points. The inertial idea was later used and developed by Nesterov [45] and Alvarez and Attouch (Refs. [4, 5]) in the sense of solving smooth convex minimization problems and monotone inclusions/non-smooth convex minimization problems respectively. A considerable amount of literature has been contributed to inertial algorithms over the last decade [2, 3, $22,56,60]$.

Fixed points iterative methods play a vital role in designing many iterative methods (Ref. [21, 10]). For example, Krasnoselskii-Mann (KM) iterative method introduced in $[42,35]$ for solving fixed point of quasi-nonexpansive mappings $T$ given by the following scheme

$$
u_{n+1}=\left(1-t_{n}\right) u_{n}+t_{n} T u_{n}, \quad n \geq 1
$$

is applied to derive iterative methods for solving convex feasibility and monotone inclusion (Refs. $[35,10,39])$. Under the condition that the sequence $t_{n} \subset[0,1]$ and $\sum_{n=1}^{\infty} t_{n}(1-$ $\left.t_{n}\right)=\infty$, the sequence generated by (1.4) is shown to converge weakly to a fixed point of the operator $T$. A more general setting of KM-type scheme that considers incorporation of inertial extrapolation step is studied in [39]. The inertial scheme is as follows:

$$
\left\{\begin{array}{l}
\bar{u}_{n}=u_{n}+\varrho_{n}\left(u_{n}-u_{n-1}\right) \\
u_{n+1}=\left(1-t_{n}\right) \bar{u}_{n}+t_{n} T \bar{u}_{n}, \quad n \geq 1 .
\end{array}\right.
$$

The convergence of the sequence generated by the scheme (1.5) is established in [39] under the assumptions that $0<\inf t_{n} \leq \sup t_{n}<1$ and $\varrho_{n} \in(0, \varrho)$, where $\varrho \in[0,1)$ and $\sum_{n=0}^{\infty} \varrho_{n}\left\|u_{n}-u_{n-1}\right\|^{2}<\infty$. In addition, iterative schemes for solving convex feasibility problem and monotone inclusion are derived from (1.5) in [39]. The scheme (1.5) is similarly studied in [10] with the strict assumptions on the parameters given as 
$0<t \leq t_{n} \leq \frac{\delta-\varrho[\varrho(1+\varrho)+\varrho \delta+\sigma]}{\delta[1+\varrho(1+\varrho)+\varrho \delta+\sigma])}$ where $\delta>\frac{\varrho^{2}(1+\varrho)+\varrho \sigma}{1-\varrho^{2}}, \sigma, t>0$ with $\{\varrho n \subset[0,1)$ is a nonincreasing sequence. Moreover, a Douglas Rachford splitting method for solving monotone inclusion problem is derived from (1.5). The observations above informed the following question.

Can we apply the KM-type scheme that considers the incorporation of inertial extrapolation step to construct a new scheme for solving variational inclusion problem (1.1)? The purpose of this paper is to give a positive answer to this question. The main contribution of this paper is to design a new scheme for solving the variational inclusion problem (1.1) as well as to derive a modified version of the proposed scheme for solving variational inequality problem and split convex feasibility problem. The proposed scheme utilizes the structure of (1.4) and the effect of inertial extrapolation step. Moreover, the proposed scheme uses self adaptive stepsize that does not involve any line search technique or does not require prior knowledge of the Lipschitz constant as in [61]. Additionally, we illustrate the potential applicability and performance of the proposed method in comparison to the existing methods in the literature by applying the proposed schemes to solve the Nash-Cournot equilibrium problem and the problem of image recovery.

In what follows, we present our propose scheme which is motivated by $[39,10,61]$.

$$
\left\{\begin{array}{l}
\bar{u}_{n}=u_{n}+\varrho_{n}\left(u_{n}-u_{n-1}\right), \\
z_{n}=\left(1-s_{n}\right) \bar{u}_{n}+s_{n}\left(I+\lambda_{n} B\right)^{-1}\left(I-\lambda_{n} A\right) \bar{u}_{n}, \\
u_{n+1}=\left(1-t_{n}\right) \bar{u}_{n}+t_{n}\left(I+\lambda_{n} B\right)^{-1}\left(I-\lambda_{n} A\right) z_{n}, \quad n \geq 1 .
\end{array}\right.
$$

where $\left\{s_{n}\right\},\left\{t_{n}\right\}$ are sequences in $(0,1)$ and $\varrho_{n}\left(u_{n}-u_{n-1}\right)$ denotes the inertial extrapolation term. The step size $\lambda_{n}$ is defined to be self-adaptively updated according to a new simple step size rule that does not depend on the Lipschitz constant of the underlying operator and does not involve any line search. The proposed scheme (1.6) can be a generalization of many important schemes, for instance, observe that, (1.6) with $\varrho_{n}=0$ and $s_{n}=0$ reduces to the KM-type scheme. Moreover, scheme (1.6) with $s_{n}=0$ reduces to the particular setting derived from KM-type scheme proposed in [39] and [10] to solve monotone inclusion problems. Additionally, (1.6) with $s_{n}=0$, and $t_{n}=1$ is a form of inertial forward-backward scheme for (1.1) proposed for example, in [38] with an identity operator as the linear self-adjoint and positive definite operator.

This work is outlined as follows: In the next Section, preliminaries consisting of lemmas, definitions and some characterizations which are essential for the convergence analysis of the proposed scheme are recalled. The main scheme and its convergence analysis are presented in Section 3. In Section 4, the computational illustrations to show the implementation and performance of the proposed scheme in comparison with some existing schemes in the literature are presented as an application in solving Nash-Cournot equilibrium problem and image deblurring problem.

\section{PRELIMINARIES}

In this section, we recall some characterizations, basic facts and lemmas that will aid in showing the convergence analysis of our proposed method. In the sequel, $\mathbb{H}$ is a real Hilbert space with the inner product $\langle\cdot, \cdot\rangle$, norm $\|\cdot\|$ and $\mathbb{E}$ is a nonempty closed and convex subset of $\mathbb{H}$. We use $u_{i} \longrightarrow u^{*}$ (resp. $\left.u_{i} \rightarrow u^{*}\right)$ to denote that, $\left\{u_{i}\right\}$ converges strongly (resp. weakly) to $u^{*}$. The following holds in a Hilbert space:

$$
\|\bar{u} \pm u\|^{2}=\|\bar{u}\|^{2}+\|u\|^{2} \pm 2\langle\bar{u}, u\rangle
$$


and

$$
\|\varrho \bar{u}+(1-\varrho) u\|^{2}=\varrho\|\bar{u}\|^{2}+(1-\varrho)\|u\|^{2}-\varrho(1-\varrho)\|\bar{u}-u\|^{2}
$$

for every $\bar{u}, u \in \mathbb{H}$.

Definition 2.1. Let a mapping $B: \mathbb{H} \longrightarrow \mathbb{H}$ be defined on a real Hilbert space $\mathbb{H}$. For all $u, v \in \mathbb{E}, B$ is said to be:

(1) Monotone if:

$$
\langle B u-B v, u-v\rangle \geq 0 .
$$

(2) Firmly nonexpansive if:

$$
\|B u-B v\|^{2} \leq\langle B u-B v, u-v\rangle,
$$

or equivalently,

$$
\|B u-B v\|^{2} \leq\|u-v\|^{2}-\|(I-B) u-(I-B) v\|^{2} .
$$

(3) $L$-Lipschitz continuous on $\mathbb{H}$ if there exists a constant $L>0$ such that:

$$
\|B u-B v\| \leq L\|u-v\| .
$$

If $L=1$, then $B$ is said to be nonexpansive.

Definition 2.2 ([9]). A multi-valued mapping $A: \mathbb{H} \longrightarrow 2^{\mathbb{H}}$ is said to be monotone, if for every $u, v \in \mathbb{H}, x \in A u$ and $y \in A v \Rightarrow\langle x-y, u-v\rangle \geq 0$. Furthermore, $A$ is said to be maximal monotone if it is monotone and if for every $(u, x) \in \mathbb{H},\langle x-y, u-v\rangle \geq 0$ for every $(v, y) \in \operatorname{Graph}(A) \Rightarrow x \in A u$.

Definition 2.3. Let $A: \mathbb{H} \longrightarrow 2^{\mathbb{H}}$ be a multi-valued maximal monotone mapping. Then, the resolvent mapping $J_{\lambda}^{A}: \mathbb{H} \longrightarrow \mathbb{H}$ associated with $A$ is defined by:

$$
J_{\lambda}^{A}(u)=(I+\lambda A)^{-1}(u),
$$

for some $\lambda>0$, where $I$ stands for the identity operator on $\mathbb{H}$.

It is worth mentioning that if $A: \mathbb{H} \longrightarrow 2^{\mathbb{H}}$ is a set-valued maximal monotone mapping and $\lambda>0$, then $\operatorname{Dom}\left(J_{\lambda}^{A}\right)=\mathbb{H}$, and $J_{\lambda}^{A}$ is a single-valued and firmly nonexpansive mapping (see. [54] for more details).

Lemma 2.1 ([11]). Let $B: \mathbb{H} \longrightarrow \mathbb{H}$ be a Lipschitz continuous and monotone mapping and $A: \mathbb{H} \longrightarrow 2^{\mathbb{H}}$ be a maximal monotone mapping, then the mapping $A+B$ is a maximal monotone mapping.

Lemma 2.2. [21] Let $\mathbb{H}$ be a real Hilbert space then for any $\tau>0$ and $\gamma \in \mathbb{R}$, we have

$$
\|u \pm \gamma v\|^{2} \geq(1-\gamma \tau)\|u\|^{2}+\gamma\left(\gamma-\frac{1}{\tau}\right)\|v\|^{2}
$$

for all $u, v \in \mathbb{H}$.

Lemma 2.3 ([47]). Suppose $\left\{\zeta_{n}\right\},\left\{\phi_{n}\right\}$ and $\left\{\varrho_{n}\right\}$ are sequences in $[0, \infty)$ such that, for all $n \geq 1$,

$$
\zeta_{n+1} \leq \zeta_{n}+\varrho_{n}\left(\zeta_{n}-\zeta_{n-1}\right)+\phi_{n}, \quad \sum \phi_{n}<\infty
$$

and there exists $\varrho \in \mathbb{R}$ with $0 \leq \varrho_{n} \leq \varrho \leq 1$ for all $n \geq 1$. Then, the following are satisfied:

(i) $\sum\left[\zeta_{n}-\zeta_{n-1}\right]_{+}<\infty$, where $[a]_{+}=\max \{a, 0\}$

(ii) there exists $\zeta^{*} \in[0, \infty)$ with $\lim \zeta_{n}=\zeta^{*}$.

Lemma 2.4. Let $\mathbb{H}$ be a nonempty closed and convex subset of a Hilbert space $\mathbb{H}$ and $T: \mathbb{E} \rightarrow \mathbb{H}$ be a nonexpansive mapping. Let $\left\{u_{n}\right\}$ be a subsequence in $\mathbb{E}$ such that $u_{n} \rightarrow u$ for $u \in \mathbb{H}$ and $\left\{u_{n}-T u_{n}\right\} \rightarrow 0$ as $n \rightarrow \infty$. Then $u \in F i x(T)$, where Fix $(T)$ denotes the set of fixed point of $T$. 
Lemma 2.5 ([48]). Let $\mathbb{E}$ be a nonempty subset of a real Hilbert space $\mathbb{H}$ and a sequence $\left\{\bar{u}_{i}\right\}$ in $\mathbb{H}$ such that:

(a) for every $\bar{u} \in \mathbb{E}, \lim _{i \rightarrow \infty}\left\|\bar{u}_{i}-u^{*}\right\|$ exists;

(b) every sequentially weak cluster point of $\left\{\bar{u}_{i}\right\}$ is in $\mathbb{E}$.

Then, $\left\{\bar{u}_{i}\right\}$ weakly converges in $\mathbb{E}$.

\section{NEW ALGORITHM FOR SOLVING VI PROBLEM}

This section presents the detail description of our proposed algorithm and the convergence analysis of the iterates generated by the algorithm which involves a maximally monotone operator $A$ and a continuous Lipschitz and monotone operator $B$. We assume the following for the analysis of the proposed method.

\section{Assumption 3.1.}

$A_{1}$ The feasible set of problem (1.1) is a nonempty closed and convex subset of $\mathbb{H}$.

$A_{2}$ The solution set $\Gamma$ of (1.1) is nonempty.

$A_{3} \quad A: \mathbb{H} \longrightarrow 2^{\mathbb{H}}$ is maximally monotone, and $B: \mathbb{H} \longrightarrow \mathbb{H}$ is L-Lipschitz continuous and monotone on $\mathbb{H}$.

$A_{4}$ Suppose the sequences $s_{n}$ and $t_{n}$ in $(0,1)$ where $0<s \leq s_{n}$ and $0<t \leq t_{n} \leq$ $\frac{\eta-\varrho(2 p \varrho(1+\varrho)+\varrho \eta(1-t)+2 p \sigma)}{\eta[1+2 p \varrho(1+\varrho)+\varrho \eta(1-t)+2 p \sigma]}$, with $\left\{\varrho_{n}\right\} \subset[0, \varrho]$ for $\varrho, \eta, p, \sigma>0$. Define the sequence

$$
\zeta_{n}=\frac{\left(1-t_{n}\right)\left(1-\varrho_{n} \tau_{n}\right)}{2 p t_{n}}
$$

and

$$
\vartheta_{n}=\varrho_{n}\left(1+\varrho_{n}\right)+\frac{\varrho_{n}\left(1-t_{n}\right)\left(1-\varrho_{n} \tau_{n}\right)}{2 p t_{n}},
$$

where $p=\left(1+\frac{1}{s}\right)$ and $\tau_{n}=\frac{1}{\varrho_{n}+\eta t_{n}}$.

\section{Algorithm 1: An Inertial Algorithm for Solving VI Problem}

Initialization: Choose $u_{-1}, u_{0} \in \mathbb{H}, \varrho_{n} \subset(0, \varrho), \varrho \in[0,1) \nu \in(0,1)$ and $\lambda_{0}>0$.

Iterative Steps: For current iterates $u_{n-1}$ and $u_{n} \in \mathbb{H}$.

Step 1. Set $\bar{u}_{n}$ as:

$$
\bar{u}_{n}=u_{n}+\varrho_{n}\left(u_{n}-u_{n-1}\right),
$$

Step 2. Compute

$$
z_{n}=\left(1-s_{n}\right) \bar{u}_{n}+s_{n}\left(I+\lambda_{n} A\right)^{-1}\left(I-\lambda_{n} B\right) \bar{u}_{n} .
$$

Step 3. Compute

$$
u_{n+1}=\left(1-t_{n}\right) \bar{u}_{n}+t_{n}\left(I+\lambda_{n} A\right)^{-1}\left(I-\lambda_{n} B\right) z_{n} .
$$

Update

$$
\lambda_{n+1}:=\left\{\begin{array}{l}
\min \left\{\frac{\nu\left\|\bar{u}_{n}-z_{n}\right\|}{\left\|B \bar{u}_{n}-B z_{n}\right\|}, \lambda_{0}\right\} \\
\lambda_{n} \text { otherwise. }
\end{array} \quad \text { if } B \bar{u}_{n} \neq B z_{n}\right.
$$

If $\bar{u}_{n}=z_{n}=u_{n+1}$ then stop and $\bar{u}_{n}$ is a solution of the VI problem, otherwise set $n:=n+1$ and go back to step 1 . 
Remark 3.1. It can be seen that the sequence $\left\{\lambda_{n}\right\}$ is monotonically decreasing. Moreover, since $A$ is a Lipschitz continuous with Lipschitz's constant $L$, for $B \bar{u}_{n} \neq B z_{n}$, we have:

$$
\frac{\nu\left\|\bar{u}_{n}-z_{n}\right\|}{\left\|B \bar{u}_{n}-B z_{n}\right\|} \geq \frac{\nu}{L}
$$

It is obvious that when $B \bar{u}_{n}=B z_{n}$ the inequality (3.11) is satisfied. Hence, it follows that $\lambda_{n} \geq \min \left\{\frac{\nu}{L}, \lambda_{0}\right\}$. Therefore, the update (3.10) is well defined.

Next, we give some important lemmas (with their proofs) that we use for the convergence analysis of the sequence generated by Algorithm 1.

Lemma 3.6. Let $\left\{\bar{u}_{n}\right\}$ be a sequence generated by Algorithm 1 and Assumption 3.1 be satisfied. For any $\breve{u} \in \Gamma$, we have

$$
\begin{aligned}
\left\|u_{n+1}-\breve{u}\right\|^{2} \leq & \left(1+\varrho_{n}\right)\left\|u_{n}-\breve{u}\right\|^{2}-\varrho_{n}\left\|u_{n-1}-\breve{u}\right\|^{2}+\varrho_{n}\left(1+\varrho_{n}\right)\left\|u_{n}-u_{n-1}\right\|^{2} \\
& -s_{n}^{2} t_{n}\left(1-t_{n}\right)\left\|\bar{u}-T_{n} z_{n}\right\|^{2} .
\end{aligned}
$$

Proof. For each $n \geq 1$. Let the resolvent $T_{\lambda_{n}}=\left(I+\lambda_{n} A\right)^{-1}\left(I-\lambda_{n} B\right)=J_{\lambda_{n}}^{A}\left(I-\lambda_{n} B\right)$, then for all $\breve{u} \in \mathbb{H}$ together with the fact that $T_{\lambda_{n}}$ is nonexpansive, we have

$$
\begin{aligned}
\left\|z_{n}-\breve{u}\right\| & =\left\|\left(1-s_{n}\right) \bar{u}_{n}+s_{n} T_{\lambda_{n}} \bar{u}_{n}-\breve{u}\right\| \\
& \leq\left(1-s_{n}\right)\left\|\bar{u}_{n}-\breve{u}\right\|+s_{n}\left\|T_{\lambda_{n}} \bar{u}_{n}-\breve{u}\right\| \\
& \leq\left(1-s_{n}\right)\left\|\bar{u}_{n}-\breve{u}\right\|+s_{n}\left\|\bar{u}_{n}-\breve{u}\right\| \\
& \leq\left\|\bar{u}_{n}-\breve{u}\right\| .
\end{aligned}
$$

On the other hand, from (3.9) and (2.8), we have

$$
\begin{aligned}
\left\|\bar{u}_{n}-\breve{u}\right\|^{2} & =\left\|\left(1+\varrho_{n}\right)\left(u_{n}-\breve{u}\right)-\varrho_{n}\left(u_{n-1}-\breve{u}\right)\right\|^{2}, \\
& =\left(1+\varrho_{n}\right)\left\|u_{n}-\breve{u}\right\|^{2}-\varrho_{n}\left\|u_{n-1}-\breve{u}\right\|^{2}+\varrho_{n}\left(1+\varrho_{n}\right)\left\|u_{n}-u_{n-1}\right\|^{2} .
\end{aligned}
$$

Similarly, from the definition of $u_{n+1}$ and Equation (2.8), we have

$$
\begin{aligned}
\left\|u_{n+1}-\breve{u}\right\|^{2} & =\left\|\left(1-t_{n}\right) \bar{u}_{n}+t_{n} T_{\lambda_{n}} z_{n}-\breve{u}\right\|^{2}, \\
& =\left(1-t_{n}\right)\left\|\bar{u}_{n}-\breve{u}\right\|^{2}+t_{n}\left\|T_{\lambda_{n}} z_{n}-\breve{u}\right\|^{2}-t_{n}\left(1-t_{n}\right)\left\|\bar{u}_{n}-T_{\lambda_{n}} z_{n}\right\|^{2} \\
& \leq\left(1-t_{n}\right)\left\|\bar{u}_{n}-\breve{u}\right\|^{2}+t_{n}\left\|z_{n}-\breve{u}\right\|^{2}-t_{n}\left(1-t_{n}\right)\left\|\bar{u}_{n}-T_{\lambda_{n}} z_{n}\right\|^{2} \\
& \leq\left(1-t_{n}\right)\left\|\bar{u}_{n}-\breve{u}\right\|^{2}+t_{n}\left\|\bar{u}_{n}-\breve{u}\right\|^{2}-t_{n}\left(1-t_{n}\right)\left\|\bar{u}_{n}-T_{\lambda_{n}} z_{n}\right\|^{2} \\
& =\left\|\bar{u}_{n}-\breve{u}\right\|^{2}-t_{n}\left(1-t_{n}\right)\left\|\bar{u}_{n}-T_{\lambda_{n}} z_{n}\right\|^{2} \\
& \leq\left\|\bar{u}_{n}-\breve{u}\right\|^{2}-s_{n}^{2} t_{n}\left(1-t_{n}\right)\left\|\bar{u}_{n}-T_{\lambda_{n}} z_{n}\right\|^{2} .
\end{aligned}
$$

The fourth and last inequality follows by substituting the estimate (3.13) and by the assumption on the sequence $s_{n}$ (Assumption $\left(A_{4}\right)$ ) respectively. Hence, the results follows by substituting (3.14) in (3.15).

Lemma 3.7. Let the solution set of the VI problem $\Gamma \neq \emptyset$ and the sequences $\left\{\vartheta_{n}\right\},\left\{\zeta_{n}\right\}$ as defined in Assumption $3.1\left(A_{4}\right)$ and $\left\{s_{n}\right\},\left\{t_{n}\right\}$ satisfy the conditions in Assumption $\left(A_{4}\right)$, then the sequence $\left\{u_{n}\right\}$ generated by Algorithm 1 satisfies

$$
Q_{n+1}-\left(1+\varrho_{n}\right) Q_{n}-\varrho_{n} Q_{n-1} \leq \zeta_{n}\left\|u_{n+1}-u_{n}\right\|^{2}+\vartheta_{n}\left\|u_{n}-u_{n-1}\right\|^{2},
$$

where $Q_{n}=\left\|u_{n}-\breve{u}\right\|^{2}$ for all $\breve{u} \in \Gamma$. 
Proof. Observe that from the definition of $z_{n}$, we have

$$
\begin{aligned}
\left\|\bar{u}_{n}-T_{\lambda_{n}} \bar{u}_{n}\right\|^{2} & =\frac{1}{s_{n}^{2}}\left\|z_{n}-\bar{u}_{n}\right\|^{2}, \\
& \geq \frac{1}{s_{n}^{2}}\left\|T_{\lambda_{n}} z_{n}-T_{\lambda_{n}} \bar{u}_{n}\right\|^{2},
\end{aligned}
$$

notice that, we have $T_{\lambda_{n}} z_{n}=\frac{1}{t_{n}}\left(u_{n+1}-\bar{u}_{n}\right)+\bar{u}_{n}$. Therefore, we have

$$
\begin{aligned}
\left\|\bar{u}_{n}-T_{\lambda_{n}} \bar{u}_{n}\right\|^{2} & =\frac{1}{s_{n}^{2}}\left\|\frac{1}{t_{n}}\left(u_{n+1}-\bar{u}_{n}\right)+\bar{u}_{n}-T_{\lambda_{n}} \bar{u}_{n}\right\|^{2}, \\
& =\frac{1}{s_{n}^{2} t_{n}^{2}}\left\|u_{n+1}-\bar{u}_{n}+t_{n}\left(\bar{u}_{n}-T_{\lambda_{n}} \bar{u}_{n}\right)\right\|^{2} .
\end{aligned}
$$

Now, using Lemma 2.2 with $\tau=\frac{1}{2 t_{n}}$ and $\gamma=t_{n}$, we get

$$
\left\|\bar{u}_{n}-T_{\lambda_{n}} \bar{u}_{n}\right\|^{2} \geq \frac{1}{s_{n}^{2} t_{n}^{2}}\left\{\frac{1}{2}\left\|u_{n+1}-\bar{u}_{n}\right\|^{2}-t_{n}^{2}\left\|\bar{u}_{n}-T_{\lambda_{n}} \bar{u}_{n}\right\|^{2}\right\} .
$$

This implies that

$$
\left(1+\frac{1}{s_{n}}\right)\left\|\bar{u}_{n}-T_{\lambda_{n}} \bar{u}_{n}\right\|^{2} \geq \frac{1}{2 s_{n}^{2} t_{n}^{2}}\left\|u_{n+1}-\bar{u}_{n}\right\|^{2},
$$

It follows from the last inequality and assumption $\left(A_{4}\right)$, that

$$
\begin{aligned}
\left(1+\frac{1}{s}\right)\left\|\bar{u}_{n}-T_{\lambda_{n}} \bar{u}_{n}\right\|^{2} & \geq \frac{1}{2 s_{n}^{2} t_{n}^{2}}\left\|u_{n+1}-\bar{u}_{n}\right\|^{2} \\
& =\frac{1}{2 s_{n}^{2} t_{n}^{2}}\left\|u_{n+1}-u_{n}-\varrho_{n}\left(u_{n}-u_{n-1}\right)\right\|^{2} .
\end{aligned}
$$

Similarly, using Lemma 2.2 as in (3.17) with $\gamma=\varrho_{n}$, we get

$$
\begin{aligned}
\left(1+\frac{1}{s}\right)\left\|\bar{u}_{n}-T_{\lambda_{n}} \bar{u}_{n}\right\|^{2} & \geq \frac{\left(1-\varrho_{n} \tau_{n}\right)}{2 s_{n}^{2} t_{n}^{2}}\left\|u_{n+1}-u_{n}\right\|^{2}+\frac{\varrho_{n}}{2 s_{n}^{2} t_{n}^{2}}\left(\varrho_{n}-\frac{1}{\tau_{n}}\right)\left\|u_{n}-u_{n-1}\right\|^{2} \\
& =\frac{\left(1-\varrho_{n} \tau_{n}\right)}{2 s_{n}^{2} t_{n}^{2}}\left\|u_{n+1}-u_{n}\right\|^{2}-\frac{\varrho_{n}\left(1-\varrho_{n} \tau_{n}\right)}{2 s_{n}^{2} t_{n}^{2} \tau_{n}}\left\|u_{n}-u_{n-1}\right\|^{2} .
\end{aligned}
$$

Multiplying both sides of (3.18) by $-s_{n}^{2} t_{n}\left(1-t_{n}\right)$, we get

Substituting the estimate (3.19) in (3.12), we have

$$
\begin{aligned}
\left\|u_{n+1}-\breve{u}\right\|^{2} \leq & \left(1+\varrho_{n}\right)\left\|u_{n}-\breve{u}\right\|^{2}-\varrho_{n}\left\|u_{n-1}-\breve{u}\right\|^{2}+\varrho_{n}\left(1+\varrho_{n}\right)\left\|u_{n}-u_{n-1}\right\|^{2} \\
& -\frac{\left(1-t_{n}\right)\left(1-\varrho_{n} \tau_{n}\right)}{2 p t_{n}}\left\|u_{n+1}-u_{n}\right\|^{2} \\
& +\frac{\varrho_{n}\left(1-t_{n}\right)\left(1-\varrho_{n} \tau_{n}\right)}{2 p t_{n} \tau_{n}}\left\|u_{n}-u_{n-1}\right\|^{2}
\end{aligned}
$$


Thus,

$$
\begin{aligned}
Q_{n+1} \leq & \left(1+\varrho_{n}\right) Q_{n}-\varrho_{n} Q_{n-1}+\varrho_{n}\left(1+\varrho_{n}\right)\left\|u_{n}-u_{n-1}\right\|^{2} \\
& -\frac{\left(1-t_{n}\right)\left(1-\varrho_{n} \tau_{n}\right)}{2 p t_{n}}\left\|u_{n+1}-u_{n}\right\|^{2} \\
& +\frac{\varrho_{n}\left(1-t_{n}\right)\left(1-\varrho_{n} \tau_{n}\right)}{2 p t_{n} \tau_{n}}\left\|u_{n}-u_{n-1}\right\|^{2},
\end{aligned}
$$

which can be simply expressed as

$$
Q_{n+1}-\left(1+\varrho_{n}\right) Q_{n}+\varrho_{n} Q_{n-1} \leq \zeta_{n}\left\|u_{n+1}-u_{n}\right\|^{2}+\vartheta_{n}\left\|u_{n}-u_{n-1}\right\|^{2},
$$

with the sequences $\zeta_{n}$ and $\vartheta_{n}$ as defined in assumption $\left(A_{4}\right)$. Hence the proof.

Lemma 3.8. Let $\left\{\vartheta_{n}\right\}$ and $\left\{\zeta_{n}\right\}$ be sequences as defined in Assumption $3.1\left(A_{4}\right)$, and the sequences $\left\{s_{n}\right\}$ and $\left\{t_{n}\right\}$ satisfy assumption $\left(A_{4}\right)$, then for all $n \geq 1$, we have

$$
\zeta_{n}+\vartheta_{n+1} \leq-\sigma_{n} .
$$

Proof. Observe that, since $\varrho_{n} \tau_{n}<1$ and $t_{n} \in(0,1)$,

$$
\varrho_{n}\left(1+\varrho_{n}\right)+\frac{\varrho_{n}\left(1-t_{n}\right)\left(1-\varrho_{n} \tau_{n}\right)}{2 p t_{n} \tau_{n}}>0 .
$$

Taking into consideration the choice $\tau_{n}=\frac{1}{\varrho_{n}+\eta t_{n}}$ in Assumption $(3.1)\left(A_{4}\right)$, we have $\eta=$ $\frac{1-\varrho_{n} \tau_{n}}{\tau_{n} t_{n}}$. Observe again that

$$
\begin{aligned}
\vartheta_{n} & =\varrho_{n}\left(1+\varrho_{n}\right)+\frac{\varrho_{n}\left(1-t_{n}\right) \eta}{2 p}, \\
& \leq \varrho(1+\varrho)+\frac{\varrho \eta(1-t)}{2 p} .
\end{aligned}
$$

Therefore,

$$
\begin{aligned}
\zeta_{n}+\vartheta_{n+1} & \leq-\sigma_{n}, \\
& \Leftrightarrow \frac{\left(1-t_{n}\right)\left(1-\varrho_{n} \tau_{n}\right)}{2 p t_{n}}+\vartheta_{n+1}+\sigma \leq 0 \\
& \Leftrightarrow\left(1-t_{n}\right)\left(1-\varrho_{n} \tau_{n}\right)+2 p t_{n}\left(\vartheta_{n+1}+\sigma\right) \leq 0 \\
& \Leftrightarrow-\left(1-t_{n}\right) \eta \tau_{n} t_{n}+2 p t_{n}\left(\vartheta_{n+1}+\sigma\right) \leq 0 \\
& \Leftrightarrow \frac{-\left(1-t_{n}\right) \eta}{\varrho_{n}+\eta t_{n}}+2 p\left(\vartheta_{n+1}+\sigma\right) \leq 0 \\
& \Leftrightarrow-\left(1-t_{n}\right) \eta+2 p\left(\vartheta_{n+1}+\sigma\right)\left(\varrho_{n}+\eta t_{n}\right) \leq 0 \\
& \Leftrightarrow 2 p\left(\vartheta_{n+1}+\sigma\right)\left(\varrho_{n}+\eta t_{n}\right)+\eta t_{n} \leq \eta .
\end{aligned}
$$

From (3.21), we get

$$
\begin{aligned}
2 p\left(\vartheta_{n+1}+\sigma\right) & \left(\varrho_{n}+\eta t_{n}\right)+\eta t_{n} \\
& \leq 2 p\left(\varrho(1+\varrho)+\frac{\varrho \eta(1-t)}{2 p}+\sigma\right)\left(\varrho_{n}+\eta t_{n}\right)+\eta t_{n} \\
& \leq \eta
\end{aligned}
$$

where the last inequality follows by using the upper bound of $\left\{t_{n}\right\}$ in assumption $\left(A_{4}\right)$. Hence the result follows.

Theorem 3.1. Suppose that Assumption 3.1 is satisfied. Then, for all $\breve{u} \in \Gamma \neq \emptyset$, the sequence $\left\{u_{n}\right\}$ generated by Algorithm 1, converges weakly to $\breve{u}$. 
Proof. Let $\breve{u} \in \Gamma$ and set $R_{n}=Q_{n}-\varrho_{n} Q_{n-1}+\vartheta_{n}\left\|u_{n}-u_{n-1}\right\|^{2}$, we make the following claims

Claim 1: $\left\{R_{n}\right\}$ is a non-increasing sequence. So, to see to that, we observe that

$$
\begin{aligned}
R_{n+1}-R_{n}= & Q_{n+1}-\varrho_{n+1} Q_{n}+\vartheta_{n+1}\left\|u_{n+1}-u_{n}\right\|^{2}-Q_{n}+\varrho_{n} Q_{n-1} \\
& -\vartheta_{n}\left\|u_{n}-u_{n-1}\right\|^{2} \\
= & Q_{n+1}-\left(1+\varrho_{n+1}\right) Q_{n}+\varrho_{n} Q_{n-1}-\vartheta_{n+1}\left\|u_{n+1}-u_{n}\right\|^{2} \\
& -\vartheta_{n}\left\|u_{n}-u_{n-1}\right\|^{2} .
\end{aligned}
$$

From Lemma 3.7, we obtain

$$
\begin{aligned}
R_{n+1}-R_{n} & \leq \zeta_{n}\left\|u_{n+1}-u_{n}\right\|^{2}+\vartheta_{n+1}\left\|u_{n+1}-u_{n}\right\|^{2}, \\
& =\left(\zeta_{n}+\vartheta_{n+1}\right)\left\|u_{n+1}-u_{n}\right\|^{2} .
\end{aligned}
$$

Now, by Lemma 3.8, the last inequality implies that for all $n \geq 1$

$$
R_{n+1}-R_{n} \leq-\sigma\left\|u_{n+1}-u_{n}\right\|^{2} .
$$

Thus, $\left\{R_{n}\right\}$ is non-increasing. Now we claim also that

Claim 2: $\sum_{n=1}^{\infty}\left\|u_{n+1}-u_{n}\right\|^{2} \leq \infty$. In fact, since $\left\{R_{n}\right\}$ is non-increasing and the sequence $\left\{\varrho_{n}\right\}$ is bounded, we have

$$
-\varrho Q_{n-1} \leq Q_{n}-\varrho Q_{n-1} \leq R_{n} \leq R_{1} .
$$

Thus, we get

$$
\begin{aligned}
Q_{n} & \leq \varrho Q_{n-1}+R_{1}, \\
& \leq \varrho\left(\varrho Q_{n-2}+R_{1}\right)+R_{1}, \\
& \vdots \\
& \leq \varrho^{n} Q_{0}+R_{1} \sum_{i=0}^{n-1} \varrho^{i} \leq \varrho^{n} Q_{0}+\frac{R_{1}}{1-\varrho} .
\end{aligned}
$$

It can be deduced from (3.23) that

$$
\begin{aligned}
\sigma \sum_{i=1}^{n}\left\|u_{n+1}-u_{n}\right\|^{2} & \leq R_{1}-R_{n+1} \\
& \leq R_{1}-\varrho Q_{n} \\
& \leq R_{1}-\varrho\left(\varrho Q_{0}+\frac{R_{1}}{1-\varrho}\right) \\
& \leq \varrho^{n+1} Q_{0}+\frac{R_{1}}{1-\varrho}
\end{aligned}
$$

Since $\varrho_{n+1} \rightarrow 0$ as $n \rightarrow \infty$, we obtain the claim. Next, we claim that

Claim 3: $\lim _{n \rightarrow \infty}\left\|u_{n}-\breve{u}\right\|$ exists and every sequential weak cluster point of sequence $\left\{u_{n}\right\}$ is in $\Gamma$. Indeed, it follows from (3.16), Claim 2 and Lemma 2.3 that, $\lim _{n \rightarrow \infty}\left\|u_{n}-\breve{u}\right\|$ exists. From Claim 2, we have

$$
\lim _{n \rightarrow \infty}\left\|u_{n+1}-u_{n}\right\|=0
$$

from the definition of $\bar{u}_{n}$, we have

$$
\begin{aligned}
\left\|\bar{u}_{n}-u_{n+1}\right\| & \leq\left\|u_{n}-u_{n+1}\right\|+\varrho_{n}\left\|u_{n}-u_{n-1}\right\|, \\
& \leq\left\|u_{n}-u_{n+1}\right\|+\varrho\left\|u_{n}-u_{n-1}\right\|,
\end{aligned}
$$


it follows from (3.24) that $\lim _{n \rightarrow \infty}\left\|u_{n+1}-\bar{u}_{n}\right\|=0$. Using the definition of $u_{n+1}$, we have

$$
\begin{aligned}
\left\|T_{\lambda_{n}} \bar{u}_{n}-\bar{u}_{n}\right\| & =\left\|T_{\lambda_{n}} \bar{u}_{n}-u_{n+1}+u_{n+1}-\bar{u}_{n}\right\|, \\
& \leq\left\|T_{\lambda_{n}} \bar{u}_{n}-u_{n+1}\right\|+\left\|u_{n+1}-\bar{u}_{n}\right\|, \\
& =\left\|T_{\lambda_{n}} \bar{u}_{n}-\left[\left(1-t_{n}\right) \bar{u}_{n}+t_{n} T_{\lambda_{n} z_{n}}\right]\right\|+\left\|u_{n+1}-\bar{u}_{n}\right\|, \\
& \leq\left\|T_{\lambda_{n}} \bar{u}_{n}-\bar{u}_{n}\right\|+t_{n}\left\|\bar{u}_{n}+T_{\lambda_{n}} z_{n}\right\|+\left\|u_{n+1}-\bar{u}_{n}\right\|, \\
& =\left\|T_{\lambda_{n}} \bar{u}_{n}-\bar{u}_{n}\right\|+t_{n}\left\|\bar{u}_{n}+T_{\lambda_{n}}\left[\left(1-s_{n}\right) \bar{u}_{n}+s_{n} T_{\lambda_{n}} \bar{u}_{n}\right]\right\|+\left\|u_{n+1}-\bar{u}_{n}\right\|, \\
& \leq\left\|T_{\lambda_{n}} \bar{u}_{n}-\bar{u}_{n}\right\|+s_{n} t_{n}\left\|\bar{u}_{n}-T_{\lambda_{n}} \bar{u}_{n}\right\|+\left\|u_{n+1}-\bar{u}_{n}\right\|, \\
& =\left(1-s_{n} t_{n}\right)\left\|T_{\lambda_{n}} \bar{u}_{n}-\bar{u}_{n}\right\|+\left\|u_{n+1}-\bar{u}_{n}\right\| .
\end{aligned}
$$

Thus,

$$
s_{n} t_{n}\left\|T_{\lambda_{n}} \bar{u}-\bar{u}\right\| \leq\left\|u_{n+1}-\bar{u}_{n}\right\| .
$$

It follows from the assumptions on the sequences $s_{n}, t_{n}$ and (3.24) that

$$
\lim _{n \rightarrow \infty}\left\|T_{\lambda_{n}} \bar{u}_{n}-\bar{u}_{n}\right\|=0 .
$$

Let $u^{*}$ be an arbitrary cluster point of $\left\{u_{n}\right\}$, then there exists a subsequence $\left\{u_{n_{i}}\right\}$ of $\left\{u_{n}\right\}$ such that $u_{n} \rightarrow u^{*} \in \mathbb{E}$. Using (3.26), it follows from Lemma 2.4 that $u^{*} \in F i x\left(T_{\lambda_{n}}\right)$ and we conclude that $u^{*} \in \Gamma$. Hence, it follows from Lemma 2.5 that $\left\{u_{n}\right\}$ converges weakly.

\section{NUMERICAL EXPERIMENTS WITH APPLICATIONS}

Here, we apply the proposed method to two main applications; we derived methods for solving variational inequality and split convex feasibility problem from the proposed method.

4.1. Application to variational inequality problem. A Variational inequality problem is a problem of finding a point $\breve{u} \in \mathbb{E}$ such that

$$
\langle B \breve{u}, u-\breve{u}\rangle \geq 0, \quad \forall u \in \mathbb{E},
$$

where $\mathbb{E}$ is a nonempty closed convex subset of a real Hilbert space $\mathbb{H}$ and $B: \mathbb{H} \longrightarrow \mathbb{H}$ is an operator. Problem (4.27) is an important problem in optimization theory with several applications in different areas of study such as economics, equilibrium, transportation, control system and so on (Ref. [7, 8, 17, 20, 29, 30, 31, 34, 36, 46, 59]).

Suppose $f: \mathbb{H} \longrightarrow(-\infty,+\infty]$ is proper lower semi-continuous and convex function. Then, for all $u \in \mathbb{H}$, the subdifferential $\partial f$ of $f$ is defined as:

$$
\partial f(u)=\{\bar{u} \in \mathbb{H}: f(u) \leq\langle\bar{u}, u-v\rangle+f(v) \quad \forall v \in \mathbb{H}\} .
$$

For a nonempty closed and convex subset $\mathbb{E}$ of $\mathbb{H}$, the indicator function $i_{\mathbb{E}}$ of $\mathbb{E}$ is given by:

$$
i_{\mathbb{E}}(u)=\left\{\begin{array}{lcc}
0 & \text { if } & u \in \mathbb{E} \\
\infty & \text { if } & u \notin \mathbb{E} .
\end{array}\right.
$$

Furthermore, the normal cone of $\mathbb{E}$ at $u, N_{\mathbb{E}} u$ is given as:

$$
N_{\mathbb{E}} u=\{\bar{u} \in \mathbb{H}:\langle\bar{u}, u-v\rangle \leq 0 \quad \forall v \in \mathbb{H}\} .
$$


It is known that the indicator function $i_{\mathbb{E}}$ is a proper lower semi-continuous and convex function on $\mathbb{H}$. Thus, the subdifferential $\partial i_{\mathbb{E}}$ of $i_{\mathbb{E}}$ is a maximal monotone operator and

$$
\begin{aligned}
\partial i_{\mathbb{E}} u & =\left\{\bar{u} \in \mathbb{H}: i_{\mathbb{E}} u \leq\langle\bar{u}, u-v\rangle+i_{\mathbb{E}} v \quad \forall v \in \mathbb{H}\right\}, \\
& =\{\bar{u} \in \mathbb{H}:\langle\bar{u}, u-v\rangle \leq 0 \quad \forall v \in \mathbb{H}\}, \\
& =N_{\mathbb{E}} u .
\end{aligned}
$$

Therefore, for all $u \in \mathbb{H}$, we can define the resolvent of $\partial i_{\mathbb{E}}$ as

$$
J_{\lambda}^{\partial i_{\mathbb{E}}}=\left(I+\lambda \partial i_{\mathbb{E}}\right)^{-1}, \text { for each } \lambda>0 .
$$

Hence, we can see that for $\lambda>0$

$$
\begin{aligned}
v=J_{\lambda}^{\partial i_{\mathbb{E}}} u & \Leftrightarrow u \in\left(v+\lambda \partial i_{\mathbb{E}} v\right), \\
& \Leftrightarrow u-v \in \lambda \partial i_{C} v, \\
& \Leftrightarrow v=P_{\mathbb{E}} u .
\end{aligned}
$$

Based on the above derivation, it has been shown that, problem (4.27) is equivalent to problem (1.1), where $A$ is a normal cone to $\mathbb{E}$ at a point $u \in \mathbb{H}$, and the resolvent $\left(I+\lambda_{n} A\right)^{-1}$ is the projection operator. In this case, we have the following result.

Theorem 4.2. Let $\mathbb{E}$ be a nonempty closed convex subset of a real Hilbert space $\mathbb{H}, B$ is a Lipschitz continuous monotone mapping on $\mathbb{H}$ and $V I(\mathbb{E}, B)$ be the solution set of the variational inequality problem (4.27). Suppose that $u_{-1}, u_{0}$ are arbitrary points in $\mathbb{H}_{1}$, and $\nu \in(0,1),\left\{\varrho_{n}\right\} \subset[0, \varrho]$ with positive numbers $\lambda_{0}, \varrho$.

$$
\left\{\begin{array}{l}
\bar{u}_{n}=u_{n}+\varrho_{n}\left(u_{n}-u_{n-1}\right), \\
z_{n}=\left(1-s_{n}\right) \bar{u}_{n}+s_{n} P_{\mathbb{E}}\left(\bar{u}_{n}-\lambda_{n} B \bar{u}_{n}\right), \\
u_{n+1}=\left(1-t_{n}\right) \bar{u}_{n}+t_{n} P_{\mathbb{E}}\left(z_{n}-\lambda_{n} B z_{n}\right),
\end{array}\right.
$$

where $P_{\mathbb{E}}$ is the metric projection onto $\mathbb{E}$ and the step size $\lambda_{n}$ is updated using Equation (3.10). If $V I(\mathbb{E}, B)$ is nonempty, then the sequence $\left\{u_{n}\right\}$ converges weakly to an element in $V I(\mathbb{E}, B)$.

As a numerical illustration, we study a generated by (4.29) Nash-Cournot oligopolistic equilibrium problem. Nash-Cournot equilibrium was reformulated as a monotone variational inequality problem in [26]. Suppose there are $n$ firms, each of them supplies a homogeneous product in a non-cooperative manner. Suppose $q_{k} \geq 0$ denotes the $k$ th firm's supply at cost $c_{k}\left(q_{k}\right)$ and $\psi=\sum_{k=1}^{n} q_{k}$ be the total supply in the market. Suppose $\varphi(\psi)$ denotes the inverse demand curve. The variational inequality problem that corresponds to this equilibrium problem is given as: find $q^{*}=\left(q_{1}^{*}, \ldots, q_{n}^{*}\right)$, such that for all $q \in \mathbb{R}_{+}^{n}$

$$
\left\langle B q^{*}, q-q^{*}\right\rangle \geq 0
$$

where $B q^{*}=\left(B\left(q_{1}^{*}\right), \ldots, B\left(q_{n}^{*}\right)\right)$, and

$$
B\left(q_{k}^{*}\right)=c_{k}^{\prime}\left(q_{k}^{*}\right)-\varphi\left(\sum_{k=1}^{n} q_{k}^{*}\right)-q_{k}^{*} \varphi^{\prime}\left(\sum_{k=1}^{n} q_{k}^{*}\right) .
$$

As a particular case, we supposed the inverse demand function $\varphi$ and the cost function $c_{k}$ be defined by:

$$
\varphi(\psi)=5000^{\frac{1}{\alpha}} \psi^{-\frac{1}{\alpha}}
$$

and

$$
c_{k}\left(q_{k}\right)=a_{k} q_{k}+\frac{\gamma_{k}}{\gamma_{k}+1} L_{k}^{\frac{1}{\gamma_{k}}} q^{\frac{\gamma_{k}+1}{\gamma_{k}}}
$$


respectively, where the constants $\alpha, a, \gamma$ and $L$ are given below. In this experiments, we consider the case $n=100$. It is important to mention that, it has been noted in [40] that many algorithms cannot be implemented for $n$ larger than 10 . We generate the data randomly for two cases, we choose each value of $\alpha, \gamma, a$ and $L$ independently from the uniform distributions with the following parameters:

i. $\alpha_{k}=1.1, \gamma_{k} \sim U\left(\frac{1}{2}, 2\right), a_{k} \sim U(1,100)$ and $L_{k} \sim U\left(\frac{1}{2}, 5\right)$.

ii. $\alpha_{k}=1.5, \gamma_{k} \sim U\left(\frac{1}{3}, 4\right), a_{k}$ and $L_{k}$ same as case $i$.

For each case above, we generate 5 random instances (the results is shown in Figure 1a and $1 \mathrm{~b}$ ). We compare the proposed method denoted as NAS with a generalized Halpern forward backward algorithm proposed in [32], this we denote by HFBF. We use $\| q-$ $P_{\mathbb{R}_{+}}(q-B q) \| \leq 10^{-6}$ as the stopping criteria and $q_{1}=(1, \ldots, 1)$ as the starting point for the comparison.

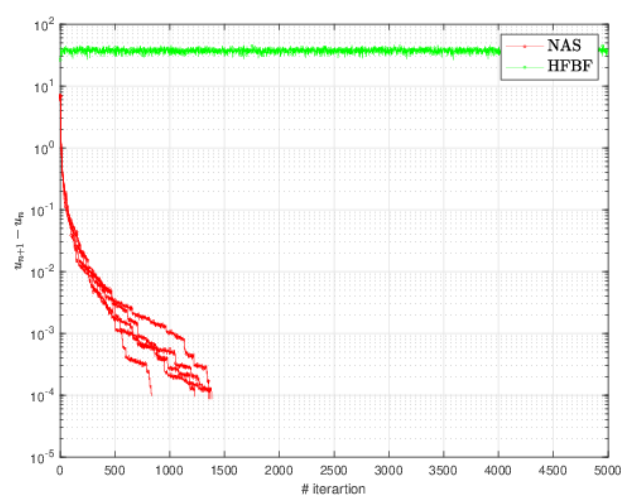

(A) case $\mathrm{i}$

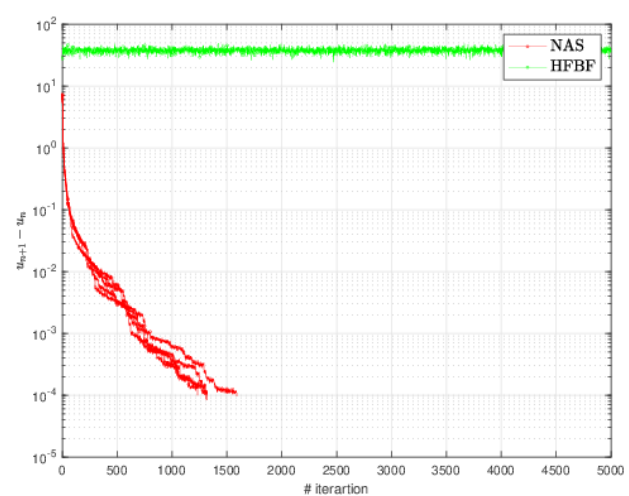

(B) case ii

FIGURE 1. Results for 5 randam instances.

From the result in Figure 1a, we can see that the proposed method performs better than the compared method in terms of number of iterations.

4.2. Application to convex feasibility problem. In this subsection, we derive a scheme for solving the split convex feasibility problem (SCFP) from Algorithm 1. The SCFP is a problem of finding a point $\check{u} \in C$ such that $B \check{u} \in Q$, where $C, Q$ are nonempty closed and convex subsets of $\mathbb{H}_{1}$ and $\mathbb{H}_{2}$, respectively, and $B: \mathbb{H}_{1} \longrightarrow \mathbb{H}_{2}$ is a bounded linear operator. Censor and Elfving [15] introduced the problem (SCFP) in finite-dimensional Hilbert spaces by using a multi-distance method to obtain an iterative method for solving SCFP. A number of problems that arise from phase retrievals, image restoration, dynamic emission, tomographic image reconstruction, radiation therapy, treatment planning, and in medical image reconstruction can be formulated as SCFP (Ref. [13, 14, 23, 12, 16] ).

Now, based on the above derivation in subsection 4.1, Algorithm 1 can be reduced to the following result.

Theorem 4.3. Let $C$ and $Q$ be nonempty closed convex subsets of Hilbert spaces $\mathbb{H}_{1}$ and $\mathbb{H}_{2}$, respectively, $B: \mathbb{H}_{1} \longrightarrow \mathbb{H}_{2}$ be a bounded linear operator with adjoint $B^{*}$, and $\Gamma_{S F P}$ be the solution set of the problem (SCFP). Let $u_{-1}, u_{0}$ be arbitrary points in $\mathbb{H}_{1}$, and $\nu \in(0,1),\left\{\varrho_{n}\right\} \subset$ $[0, \varrho]$ with positive numbers $\lambda_{0}, \varrho$. 


$$
\left\{\begin{array}{l}
\bar{u}_{n}:=u_{n}+\varrho_{n}\left(u_{n}-u_{n-1}\right), \\
z_{n}:=\left(1-s_{n}\right) \bar{u}_{n}+s_{n} P_{C}\left[\bar{u}_{n}-\lambda_{n} B^{*}\left(I-P_{Q}\right) B \bar{u}_{n}\right], \\
u_{n+1}:=\left(1-t_{n}\right) \bar{u}_{n}+t_{n} P_{C}\left[z_{n}-\lambda_{n} B^{*}\left(I-P_{Q}\right) B z_{n}\right] .
\end{array}\right.
$$

where the step size $\lambda_{n}$ is updated using Equation 3.10. If $\Gamma_{S F P} \neq \emptyset$, then the sequence $\left\{u_{n}\right\}$ converges weakly to an element of $\Gamma_{S F P} \neq \emptyset$.

In what follows, we implement Algorithm 1 and the derived scheme (4.30) in solving image deblurring problem. Furthermore, to illustrate the effectiveness of the proposed schemes, we give a comparative analysis of Algorithm 1 and the algorithms proposed in [38] and [44].

Recall that the image deblurring problem in image processing can be expressed as:

$$
c=M u+\delta
$$

where $u \in \mathbb{R}^{n}$ represents the original image, $M$ is the deblurring matrix, $c$ is the observed image, and $\delta \in \mathbb{R}^{m}$ is the Gaussian noise. It has been known that solving (4.31) is equivalent to solving the convex unconstrained optimization problem:

$$
\min _{u \in \mathbb{R}^{n}} f(u):=\frac{1}{2}\|M u-c\|_{2}^{2}+\rho\|u\|_{1}^{2}
$$

with $\rho>0$ as the regularization parameter. To solve (4.32), we suppose $B=\nabla S(u)$ and $A=\partial T$ where $S(u)=\frac{1}{2}\|M u-c\|_{2}^{2}$ and $T(u)=\|u\|_{1}^{2}$, then we have $\nabla S(u)=M^{t}(M u-c)$ is $\frac{1}{\|M\|^{2}}$-cocoercive. Therefore, for any $0<\tau<\frac{2}{\|M\|^{2}},(I-\tau \nabla S)$ is nonexpansive [28]. The subgradient $\partial T$ is maximal monotone [52]. It is well known that:

$$
u \text { is a solution of }(4.32) \Leftrightarrow 0 \in(A+B) u \Leftrightarrow u=\operatorname{prox}_{\rho T}(I-\tau \nabla S)(u)
$$

where $\operatorname{prox}_{\rho T}(u)=\arg \min _{x \in \mathbb{R}^{n}}\left\{T(u)+\frac{1}{2 \rho}\|u-x\|^{2}\right\}$. For more details, see [18].

To measure the quality of the recovered images, we adopted the improved signal-tonoise ratio (ISNR) [49] and structural similarity index measure (SSIM) [62]. We considered motion blur from MATLAB as the blurring function using ("fspecial('motion', 9, 40)"). For the comparison, we considered the standard test images of Girl $(768 \times 512)$, Fruits $(512 \times 512)$, and Tulips $(768 \times 512)($ see Figure $2 \mathrm{a}, 2 \mathrm{~b}$ and $2 \mathrm{c})$. For the control parameters, we took $\varrho=0.9, \lambda_{0}=1, \nu=0.3$, and $\rho=0.1$, for Algorithm 1 . While for the compared algorithms (Algorithm 3.1, [38] and Algorithm 1.3, [44]) the control parameters are set as reported in their respective papers. For all algorithms, we took $\frac{\left\|u_{n+1}-u_{n}\right\|_{2}}{\left\|u_{n+1}\right\|_{2}}<10^{-4}$ as the stopping criterion. For reference, all codes were written using MATLAB R2019b on a personnel computer. 


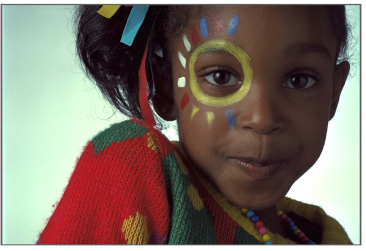

(A) Girl

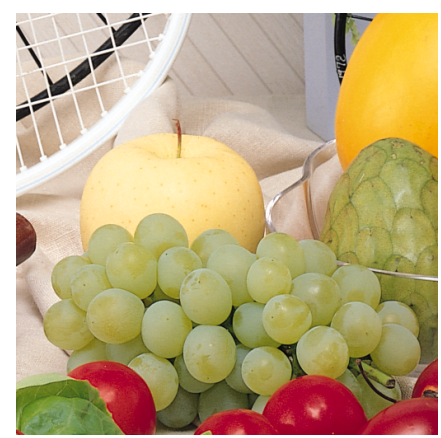

(B) Fruits

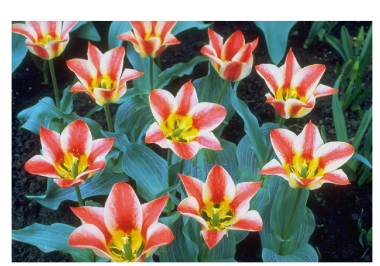

(C) Tulips

FIGURE 2. Original test images.

TABLE 1. The ISNR and SSIM values of the compared algorithms

\begin{tabular}{|c|c|c|c|c|c|c|}
\hline \multirow[b]{2}{*}{ Images } & \multicolumn{2}{|c|}{ Algorithm 1} & \multicolumn{2}{|c|}{ Algorithm 1.3} & \multicolumn{2}{|c|}{ Algorithm 3.1} \\
\hline & ISNR & SSIM & ISNR & SSIM & ISNR & SSIM \\
\hline Girl & 5.7228 & 0.9277 & 5.5838 & 0.9267 & 5.5743 & 0.9267 \\
\hline Fruits & 6.2731 & 0.9442 & 6.0838 & 0.9430 & 6.0753 & 0.9429 \\
\hline Tulips & 7.3830 & 0.9167 & 7.1361 & 0.9145 & 7.1235 & 0.9144 \\
\hline
\end{tabular}

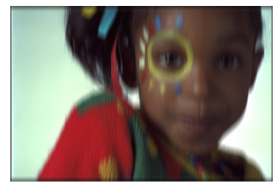

(A) degraded

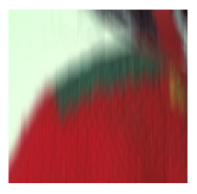

(E) degraded

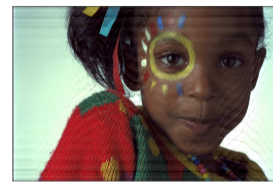

(B) Algorithm 1.0

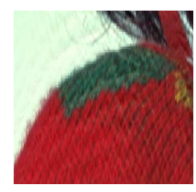

(F) Algorithm 1.0

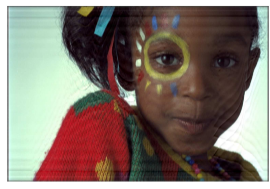

(c) Algorithm 3.1

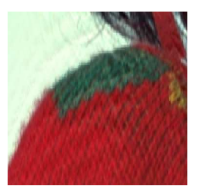

(G) Algorithm 3.1

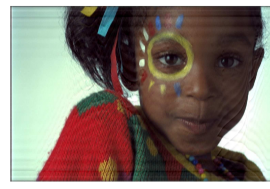

(D) Algorithm 1.3

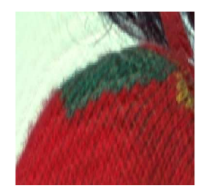

(H) Algorithm 1.3

FIGURE 3. Degraded and restored Girl images by the various algorithms. 


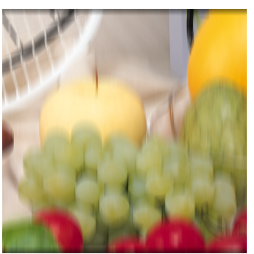

(A) degraded

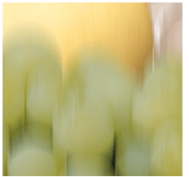

(E) degraded

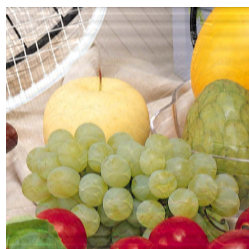

(B) Algorithm 1.0

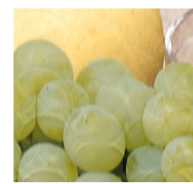

(F) Algorithm 1.0

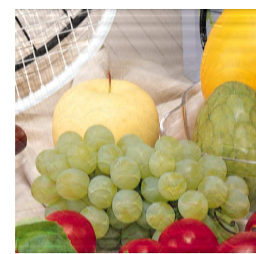

(C) Algorithm 3.1

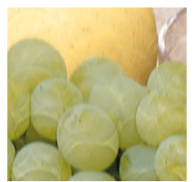

(G) Algorithm 3.1

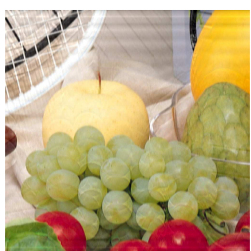

(D) Algorithm 1.3

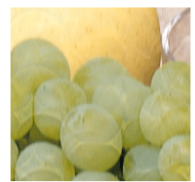

(H) Algorithm 1.3

FIGURE 4. Degraded and restored Fruits images by the various algorithms.

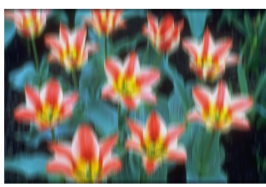

(A) degraded

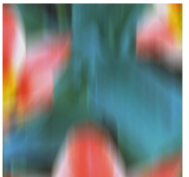

(E) degraded

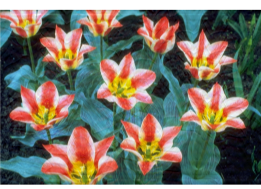

(B) Algorithm 1.0

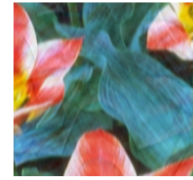

(F) Algorithm 1.0

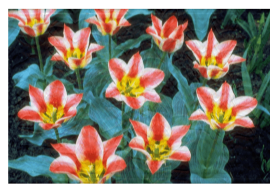

(C) Algorithm 3.1

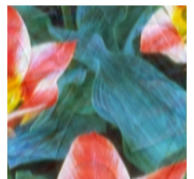

(G) Algorithm 3.1

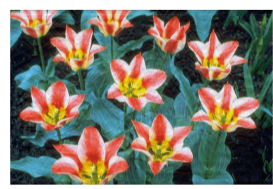

(D) Algorithm 1.3

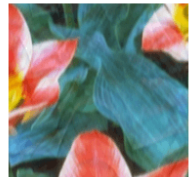

(H) Algorithm 1.3

FIGURE 5. Degraded and restored Tulips images by the various algorithms.

It can be seen from Table 1 that the recovered images (Figures $3 b, 3 f, 4 b 4 f, 5 b$ and $5 \mathrm{f}$ in 3, 4 and 5 respectively) by Algorithm 1 have higher ISNR and SSIM values, which means that the quality of images recovered by Algorithm 1 is better than the compared algorithms.

For the split convex feasibility problem (SCFP), we reformulate Problem 4.32 as a convex constrained optimization problem:

$$
\begin{aligned}
& \min _{u \in \mathbb{R}^{n}} \frac{1}{2}\|M u-c\|_{2}^{2} \\
& \text { subject to }\|u\|_{1} \leq t,
\end{aligned}
$$

where $t>0$ is a given constant, and to solve (4.33), we take $B u=\nabla S(u)$ and consider $C:=\left\{u \in \mathbb{R}^{n}:\|u\|_{1} \leq t\right\}$ and $Q:=\{c\}$.

We compare scheme 4.30 (Algorithm 2.0) with Byrne's algorithm proposed in [12] (Algorithm 1.1) for solving the SCFP. We take the same control parameters and stopping 
criteria as in problem (4.32) above for Algorithm 2.0 and for Algorithm 1.1 in [12] we take $\lambda=0.5$ and $\gamma=\frac{0.7}{\|B\|^{2}}$.

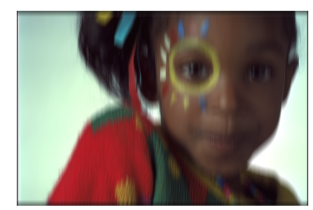

(A) degraded

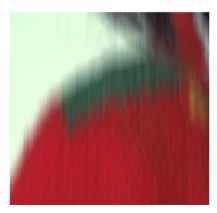

(D) degraded

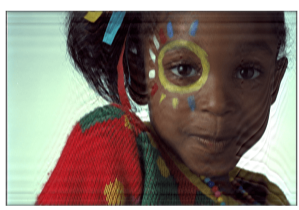

(B) Algorithm 2.0

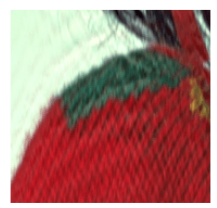

(E) Algorithm 2.0

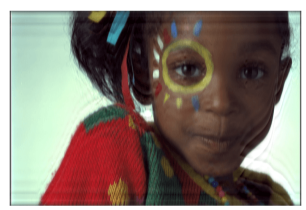

(C) Algorithm 1.1

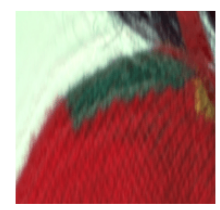

(F) Algorithm 1.1

FIGURE 6. Degraded and restored Girl images by the various algorithms.

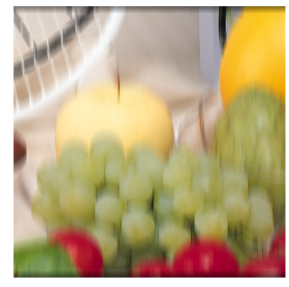

(A) degraded

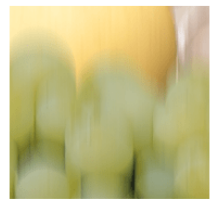

(D) degraded

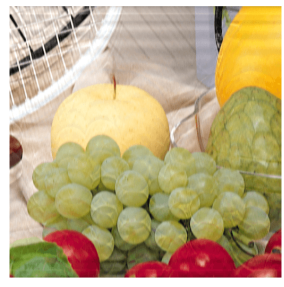

(B) Algorithm 2.0

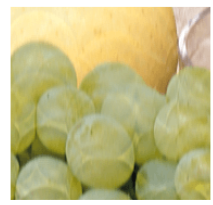

(E) Algorithm 2.0

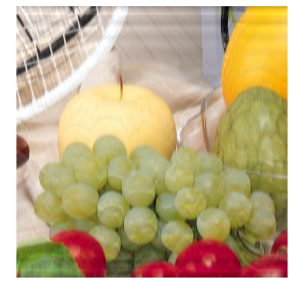

(c) Algorithm 1.1

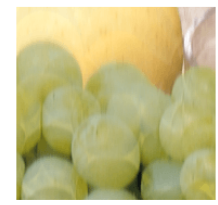

(F) Algorithm 1.1

FIGURE 7. Degraded and restored Fruits images by the various algorithms. 


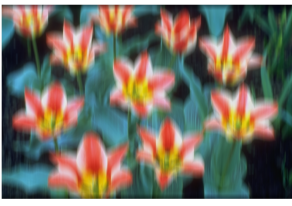

(A) degraded

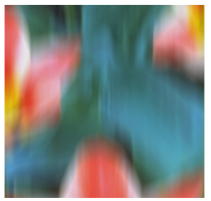

(D) degraded

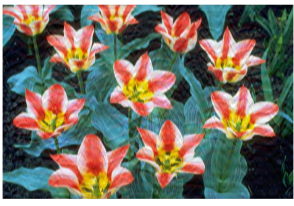

(B) Algorithm 2.0

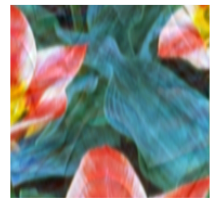

(E) Algorithm 2.0

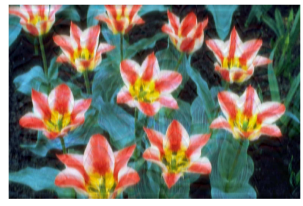

(C) Algorithm 1.1

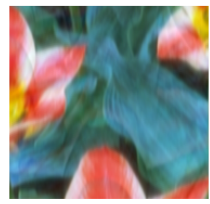

(F) Algorithm 1.1

FIGURE 8. Degraded and restored Tulips image by the various algorithms.

Figures 6, 7, 8 and Table 2 below shows that the recovered images by our algorithm (Algorithm 2) have higher ISNR and SSIM values, which means that the quality of images recovered by Algorithm 2.0 is better compared to Algorithm 1.1 of Byrne [12].

TABLE 2. The ISNR and SSIM values of the compared algorithms.

\begin{tabular}{cllll}
\hline & \multicolumn{2}{c}{ Algorithm 2.0 } & \multicolumn{2}{c}{ Algorithm 1.1 } \\
\cline { 2 - 5 } Images & ISNR & SSIM & ISNR & SSIM \\
\hline Girl & 4.9823 & 0.9150 & 4.0472 & 0.9117 \\
Fruits & 5.3341 & 0.9324 & 4.3100 & 0.9269 \\
Tulips & 6.1536 & 0.8923 & 4.9314 & 0.8853 \\
\hline
\end{tabular}

\section{CONCLUSIONS}

A new accelerated method for solving variational inclusion problems is proposed in this work, and the scheme was derived by incorporating the inertial extrapolation step with a generalized KM-type method. The main advantage of this scheme is that it involves both the use of an inertial extrapolation step and relaxation technique, which makes the iterates generated by the proposed scheme to converge weakly to the solution of the zeros of the sum of a maximally monotone operator and a monotone operator. Furthermore, the proposed method does not require prior knowledge of the Lipschitz constant of the underlying operator, and the numerical experiments suggest that the iterates generated by the proposed scheme converges fast to the solution of the problem due to the combination of the inertial extrapolation step and the relaxation technique. A modified schemes derived from the proposed method were given for solving variational inequality and split feasibility problem. The application of the proposed methods in solving the Nash-Cournot equilibrium problem and image deblurring problem, and the comparison with some of the related methods in the literature suggest that the proposed methods are robust and efficient.

Acknowledgement. The authors acknowledge the financial support provided by the Center of Excellence in Theoretical and Computational Science (TaCS-CoE), KMUTT. The first 
authors was supported by Petchra Pra Jom Klao Ph.D. Research Scholarship from King Mongkut's University of Technology Thonburi, Thailand (Grant Nos. 38/2018). Moreover, this research was funded by Thailand Science Research and Innovation Fund, and King Mongkut's University of Technology North Bangkok with Contract no. KMUTNBBasicR-64-22.

\section{REFERENCES}

[1] Abubakar, J.; Kumam, P.; Ibrahim, A. H.; Padcharoen, A. Relaxed inertial Tseng's type method for solving the inclusion problem with application to image restoration. Mathematics 8 (2020), no. 5, 818 .

[2] Abubakar, J.; Kumam, P.; Rehman, H.; Ibrahim, A. H. Inertial iterative schemes with variable step sizes for variational inequality problem involving pseudomonotone operator. Mathematics 8 (2020), no. 4, 609.

[3] Abubakar, J.; Sombut, K.; Rehman, H.; Ibrahim, A. H. An accelerated subgradient extragradient algorithm for strongly pseudomonotone variational inequality problems. Thai J. Math. 18 (2019), no. 1.

[4] Alvarez, F. On the minimizing property of a second order dissipative system in hilbert spaces. SIAM J. Control Optim. 38 (2000), no. 4, 1119-2000.

[5] Alvarez, F.; Attouch, H. An inertial proximal method for maximal monotone operators via discretization of a nonlinear oscillator with damping. Set-Valued Analysis 9 (2001), no. 1-2, 3-11.

[6] Attouch, H.; Peypouquet, J.; Pedont, P. An Backward-forward algorithms for structured monotone inclusions in hilbert spaces. J. Math. Anal. Appl. 457 (2018), no. 2, 1095-1117.

[7] Aubin, J. P.; Ekeland, I., Applied Nonlinear Analysis, John Wiley and Sons, New York, 1984.

[8] Baiocchi, C., Variational and quasivariational inequalities-Applications to free-boundary problems. John Wiley, New York, 1984.

[9] Bauschke H. H.; Combettes, P. L. Convex analysis and monotone operator theory in Hilbert spaces. Springer 408 2011.

[10] Bot, R. I.; Csetnek, R. E.; Hendrich, C. Inertial douglas-rachford splitting for monotone inclusion problems. Appl. Math. Comput 256 (2015), 472-487.

[11] Brezis, H. Operateurs Maximaux Monotones et semi-groupes de contractions dans les espaces de Hilbert. Elsevier 1973.

[12] Byrne, C. Iterative oblique projection onto convex sets and the split feasibility problem. Inverse problems $\mathbf{1 8}$ (2002), no. 2, 441.

[13] Byrne, C. A unified treatment of some iterative algorithms in signal processing and image reconstruction. Inverse problems 20 (2003), no. 1, 103.

[14] Censor, Y.; Bortfeld, T.; Martin, B.; Trofimov, A. A unified approach for inversion problems in intensitymodulated radiation therapy. Physics in Medicine E Biology 51 (2006), no. 10, 2353.

[15] Censor, Y.; Elfving, T. A multiprojection algorithm using bregman projections in a product space. Numerical Algorithms 8 (1994), no. 2, 221-239.

[16] Censor, Y.; Elfving, T.; Kopf, N.; Bortfeld, T. The multiple-sets split feasibility problem and its applications for inverse problems. Inverse Problems 21 (2005), no. 6, 2071.

[17] Chamnarnpan, T.; Phiangsungnoen, S.; Kumam, P. A new hybrid extragradient algorithm for solving the equilibrium and variational inequality problems. Afrika Matematika 26 (2015), no. 1-2, 87-98.

[18] Combettes, P. L.; RWajs, V. Signal recovery by proximal forward-backward splitting. Multiscale Modeling $\mathcal{E}$ Simulation 4 (2005), no. 4, 1168-1200.

[19] Dadashi, V.; Postolache, M. Forward-backward splitting algorithm for fixed point problems and zeros of the sum of monotone operators. Arab. J. Math. (Springer) 9 2020, No. 1, 89-99.

[20] Deepho, J.; Kumam, W.; Kumam, P. A new hybrid projection algorithm for solving the split generalized equilibrium problems and the system of variational inequality problems. J. Math. Model. Algorithms Oper. Res. 13 (2014), no. 4, 405-423.

[21] Dixit, A.; Sahu, D. R.; Singh, A. K.; Som, T. Application of a new accelerated algorithm to regression problems. Soft Computing 24 (2020), no. 2, 1539-1552.

[22] Dong, Q. L.; Cho, Y. J.; Zhong, L. L.; Rassias, Th. M. Inertial projection and contraction algorithms for variational inequalities. J. Glob. Optim 70 (2018), no. 3, 687-704.

[23] Gibali, A.; Thong, V. T. Tseng type methods for solving inclusion problems and its applications. Calcolo 55 (2018), no. 4, 49.

[24] Goldstein, A. A. Convex programming in Hilbert space. Bull. Amer. Math. Soc. 70 (1964), no. 5 709-710.

[25] Hanjing, A.; Suantai, S. A fast image restoration algorithm based on a fixed point and optimization method. Mathematics 8 (2020), no. 3, 378.

[26] Harker, P. T. A variational inequality approach for the determination of oligopolistic market equilibrium. Mathematical Programming 30 (1984), no. 1, 105-111. 
[27] Huang, Y.; Dong Y. New properties of forward-backward splitting and a practical proximal-descent algorithm. Appl. Math. Comput. 237 (2014), 60-68.

[28] Iiduka, H.; Takahashi, W., Strong convergence theorems for nonexpansive nonself-mappings and inversestrongly-monotone mappings. J. Convex Anal. 11 (2004), no. 1, 69-79.

[29] Jaiboon, C.; Kumam, P. An extragradient approximation method for system of equilibrium problems and variational inequality problems. Thai J. Math., 7 (2012), no. 1, 77-104.

[30] Khobotov, E. N. Modification of the extra-gradient method for solving variational inequalities and certain optimization problems. USSR Comput. Math. \& Math. Phys. 27 (1987), no. 5, 120-127.

[31] Kinderlehrer, D.; Stampacchia, G. An introduction to variational inequalities and their applications. Siam. 31 (1980), no. 10.

[32] Kitkuan, D.; Kumam, P.; Moreno, J. M. Generalized Halpern-type forward-backward splitting methods for convex minimization problems with application to image restoration problems, Optimization, 2019, 1-25.

[33] Kitkuan, D.; Kumam, P.; Moreno, J. M.; Sitthithakerngkiet, K. Inertial viscosity forward-backward splitting algorithm for monotone inclusions and its application to image restoration problems. Int. J. Comput. Math., 97 (2020), no. 1-2, 482-497.

[34] Konnov, I. Combined relaxation methods for variational inequalities, Springer Science \& Business Media 495 2001.

[35] Krasnosel'ski1, M. A. Two remarks on the method of successive approximations. Uspekhi Matematicheskikh Nauk 10 (1955), no. 1, 63.

[36] Kumam, W., Piri, H.; Kumam, P., Solutions of system of equilibrium and variational inequality problems on fixed points of infinite family of nonexpansive mappings, Appl. Math. Comput. 248 (2014), 441-455.

[37] Lions, P. L.; Mercier, B. Splitting algorithms for the sum of two nonlinear operators. SIAM J. Numer. Anal. 16 (1979), no. 6, 964-979.

[38] Lorenz D. A.; Pock T. An inertial forward-backward algorithm for monotone inclusions. J. Math. Imaging Vis. 51 (2015), no. 2, 311-325.

[39] Maingé P. E., Convergence theorems for inertial km-type algorithms, Computational and Applied Mathematics, 219 (2008), no. 1, 223-236.

[40] Malitsky Y. Golden ratio algorithms for variational inequalities. Mathematical Programming (2019), 1-28.

[41] Malitsky, Y.; Tam, M. K. A forward-backward splitting method for monotone inclusions without cocoercivity. SIAM J. Control Optim. 30 (2020), no. 2, 1451-1472.

[42] Mann, W. R. Mean value methods in iteration, in Proceedings of the American Mathematical Society, 1953 SIAM J. Control Optim. 4 (2020), no. 3, 506-510.

[43] Marcotte, P. Application of khobotov's algorithm to variational inequalities and network equilibrium problems. INFOR: Information Systems and Operational Research 29 (1991), no. 4, 258-270.

[44] Moudafi, A.; Oliny, M. Convergence of a splitting inertial proximal method for monotone operators. J. Comput. Appl. Math. 155 (2003), no. 2, 447-454.

[45] Nesterov, Y. A method for unconstrained convex minimization problem with the rate of convergence o $\left(\frac{1}{k^{2}}\right)$. Doklady an ussr 269 1984, 543-547.

[46] Noor, M. A. Some developments in general variational inequalities. Appl. Math. Comput. 152 (2004), no. 1, 199-277.

[47] Ofoedu, E. U. Strong convergence theorem for uniformly L-Lipschitzian asymptotically pseudocontractive mapping in real Banach space J. Math. Anal. Appl., 321 (2006), no. 2, 722-728.

[48] Opial, Z. Weak convergence of the sequence of successive approximations for nonexpansive mappings. Bull. Am. Math. Soc. 73 (1967), no. 4, 591-597.

[49] Padcharoen, A.; Kitkuan, D.; Kumam, W.; Kumam, P., Tseng methods with inertial for solving inclusion problems and application to image deblurring and image recovery problems. Comput. Math. Methods, (2020), 1088.

[50] Passty, G. B. Ergodic convergence to a zero of the sum of monotone operators in Hilbert space. J. Math. Anal. Appl. 72 (1979), no. 2, 383-390.

[51] Polyak, B. T. Some methods of speeding up the convergence of iteration methods. USSR Comput. Math. $\mathcal{E}$ Math. Phys. 4 (1964), no. 5, 1-17.

[52] Rockafellar, R. T. Monotone operators and the proximal point algorithm SIAM J. Control Optim 14 (1976), no. $5,877-898$.

[53] Suparatulatorn, R.; Khemphet, A. Tseng type methods for inclusion and fixed point problems with applications. Mathematics 7 (2019), no. 12, 1175.

[54] Takahashi, W. Nonlinear functional analysis-fixed point theory and its applications, Yokohama Publishers, 2000.

[55] Thong, D. V.; Cholamjiak, P. Strong convergence of a forward-backward splitting method with a new step size for solving monotone inclusions. Comput. Appl. Math. 38 (2019), no. 2, Paper No. 94, 16 pp. 
[56] Thong, D. V.; Hieu, D. V. Inertial extragradient algorithms for strongly pseudomonotone variational inequalities. J. Comput. Appl. Math. 341 (2018), 80-98.

[57] Trémolières R.; Lions, J. L.; Roland, G. Numerical analysis of variational inequalities. Elsevier Publishers, 2011.

[58] Tseng, P. A modified forward-backward splitting method for maximal monotone mappings. SIAM J. Control Optim. 38 (2000), no. 2, 431-446.

[59] Rehman ur., H.; Kumam, P.; Cho, Y. J.; Yordson, P. Weak convergence of explicit extragradient algorithms for solving equilibirum problems. J. Inequal Appl. 2019 (2019), no. 1, 1-25.

[60] Hieu, D. V. An inertial-like proximal algorithm for equilibrium problems. Math. Methods Oper. Res. 88 (2018), No. 3, 1-17.

[61] Hieu, D. V.; Anh P. K.; Muu, L. D. Modified forward-backward splitting method for variational inclusions. 4OR Q. J. Oper. Res. (2020), 1-25.

[62] Wang, Z.; Bovik, A. C.; Sheikh, H. R.; Simoncelli, E. P. Image quality assessment: from error visibility to structural similarity. IEEE Trans. Image Process 13 (2004), no. 4, 600-612.

DEPARTMENT OF MATHEMATICS

King MONGKUT'S UNIVERSITY OF TECHNOLOGY THONBURI (KMUTT)

126 Pracha-Uthit RoAd, 10140, BAng Mod, Thrung KHrU, BANGKoK, Thailand

Email address: poom.kum@kmutt.ac.th, ibrahimkarymegmail.com

DEPARTMENT OF MATHEMATICS

USMANU DANFODIYO UNIVERSITY SOKOTO

P. M. B. 2346, SOKOTO, NigERIA

Email address: garba.isa@udusok.edu.ng

Email address: abubakar. jamilu@udusok.edu.ng

Email address: abdullahi.sirajo@udusok.edu.ng

DEPARTMENT OF MATHEMATICS

FACULTY OF APPLIED SCIENCE

INTELLIGENT AND NONLINEAR DYNAMIC INNOVATIONS RESEARCH CENTER

King MONGKUT'S University OF TECHNOLOGY NORTH BANGKOK (KMUTNB), BANGKOK, THAILAND

Email address: kanokwan.sesci.kmutnb.ac.th 